\title{
THE WEAKLY COMPLEX BORDISM OF LIE GROUPS
}

\author{
BY TED PETRIE
}

Communicated by William Browder, May 1, 1967

1. Preliminaries. Let $\Re$ be the class of compact 1 connected semisimple Lie groups; $\mathfrak{K}^{\prime} \subset \mathfrak{K}$ is the following set of groups, $\operatorname{Sp}(n), \mathrm{SU}(n)$, $\operatorname{Spin}(n), G_{2}, F_{4}, E_{6}, E_{7}, E_{8}, U_{*}(X)$ the weakly complex bordism of $X[1]$ and $\Lambda$ the ring $U_{*}(p t)=Z\left[Y_{1}, Y_{2}, \cdots\right] . \Lambda$ is the weakly complex bordism ring defined by Milnor. The generators $Y_{i}$ are weakly complex manifolds of dim $2 i$. The bordism class of a weakly complex manifold $M^{2 n}$ is determined by its Milnor numbers [2] $s_{\omega}\left[M^{2 n}\right]$ for $\omega$ ranging over all partitions of $n$. In particular, the generators $Y_{i}$ can be chosen so that $s_{i}\left(Y_{i}\right)=1$ unless $i=p^{k}-1$ for some prime $p$ and in this case $s_{i}\left(Y_{i}\right)=p$; moreover, we assume generators $Y_{i}$ chosen so that its Todd genera are 1.

It is possible and convenient to introduce bordism theories with other coefficient rings than $\Lambda$. If $\Gamma$ is such a ring, $U_{*}(, \Gamma)$ will denote the resulting theory. Briefly here are some examples: $\Lambda_{p}$ $=Z_{p}\left[Y_{1}, Y_{2}, \cdots\right], \Lambda\left[1 / Y_{p-1}\right]=\operatorname{direct} \lim 1 / Y_{p-1}^{n} \Lambda$ and $\Lambda_{p}\left[1 / Y_{p-1}\right]$ $=\operatorname{direct} \lim 1 / Y_{p-1}^{n} \Lambda_{p}{ }^{1}$ Let $M=\left\{M_{n}\right\}$ denote the stable object of Milnor [1] and $Z_{p}=S^{1} U_{p} E^{2}$ the space obtained by attaching $E^{2}$ to $S^{1}$ via a map of degree $p . M_{n+2}^{Z_{p}}$ denotes the space of base point preserving maps from $Z_{p}$ to $M_{n+2}$. Then $U_{k}\left(X, \Lambda_{p}\right)=\operatorname{direct} \lim \Pi_{n+k}\left(X^{+} \wedge M_{n+2}^{z_{p}}\right)$ $X^{+}$is the disjoint union of $X$ and a point $x_{0} \cdot U_{*}\left(X, \Lambda_{p}\right)$ is the resulting theory. $U_{*}\left(X, \Lambda\left[1 / Y_{p-1}\right]\right)=U_{*}(X) \otimes_{\Lambda} \Lambda\left[1 / Y_{p-1}\right]$ and $U_{*}(X$, $\left.\Lambda_{p}\left[1 / Y_{p-1}\right]\right)=U_{*}\left(X, \Lambda_{p}\right) \otimes_{\Lambda p} \Lambda_{p}\left[1 / Y_{p-1}\right]$.

To $K \subset \mathfrak{K}$ there is associated a "generating variety" $K_{s}$ introduced by Bott [4]. Essentially $K_{s}$ is the homogeneous space $K / K^{s}$ where $K^{s}$ is the centralizer of a 1-dimensional torus $S^{1} \subset K$. The dimension of the center of $K^{s}$ is 1 . The commutator map

$$
S^{1} \times K_{\mathrm{s}} \stackrel{[]]}{\longrightarrow} K
$$

defined by $[t,[k]]=t k t^{-1} k^{-1}$ for $[k] \in K_{s}, t \in S^{1} \subset K$ is of particular importance.

2. Statement of results. Define $\Lambda(K)=\Lambda$ if $H^{*}(K)$ has no torsion, $=\Lambda\left[1 / Y_{1}\right]$ if $H^{*}(K)$ has only 2 torsion, $=\Lambda\left[1 / Y_{1}, 1 / Y_{2}\right]$ if $H^{*}(K)$ has only 2,3 torsion, $=\Lambda\left[1 / Y_{1}, 1 / Y_{2}, 1 / Y_{4}\right]$ if $H^{*}(K)$ has 2,3 and 5 torsion.

\footnotetext{
1 E.g., $\Lambda\left[1 / Y_{p-1}\right]$ is the ring obtained from $\Lambda$ by making $Y_{p-1}$ a unit.
} 
Theorem 1. If $K=\operatorname{Spin}(n), \mathrm{Sp}(n), \mathrm{SU}(n)$ or $G_{2}, \mathrm{Im}[]_{*}$ generates $U_{*}(K, \Lambda(K))$ and $E_{0} U_{*}(K, \Lambda(K))$ is an exterior algebra on rank $K$ generators for some filtration of $U_{*}(K, \Lambda(K))$.

Theorem 2. If $K \subset \mathcal{K}^{\prime}$ then $\operatorname{Im}[]_{p *}$ generates $U_{*}\left(K, \Lambda_{p}\left[1 / Y_{p-1}\right]\right)$ and $E_{0} U_{*}\left(K, \Lambda_{p}\left[1 / Y_{p-1}\right]\right)$ is an exterior algebra on rank $K$ generators (except possibly for $\left.\left(E_{7}, 2\right),\left(E_{8}, 2\right),\left(E_{8}, 3\right)\right) . p$ is a prime.

Corollary 3. If $K \subset K^{\prime}$, Im [ ]* generates (algebraically) $U_{*}\left(K, \Lambda(K)\right.$ ) and $U_{*}(K, \Lambda(K))$ is a torsion free abelian group (except possibly for $E_{7}$ and $\left.E_{8}\right)$.

Corollary 4 (Hodgkin). For $K$ as in Theorem $1, K^{*}(K)$ is an exterior algebra on rank $K$ generators.

THEOREM 5. For $n \geqq 7 U_{*}(\operatorname{Spin}(n))$ has 2 torsion and $Y_{1}$ torsion

Theorem 6. For any $i$, the $Y_{i}$ torsion subgroup of $U_{*}(\operatorname{Spin}(n))$ is contained in the $Y_{1}$ torsion subgroup of $U_{*}(\operatorname{Spin}(n))$.

3. Outline of techniques. The most significant fact about the $K \subset \varkappa$ is that the homology of $G=\Omega K$ is all even dimensional and generated by weakly complex manifolds [4], [5]. The method we have chosen to exploit this fact is the following: The Milnor construction of the classifying space $K$ of $\Omega K$ leads to a spectral sequence converging to $U_{*}(K)$ [6]. The $E^{2}$ term in this case is Tor $U_{*}(G)(\Lambda, \Lambda)$ because $U_{*}(G)$ is $\Lambda$ free. (This follows from the fact.) Introducing $\Gamma$ coefficients, there results a spectral sequence $\operatorname{Tor}_{*}^{U_{*}(G, \Gamma)}(\Gamma, \Gamma)$ $\Rightarrow U_{*}(K, \Gamma)$. The ring $U_{*}(G, \Gamma)$ is determined for various $\Gamma$. $\operatorname{Tor}^{U *(G, \Gamma)}(\Gamma, \Gamma)$ is shown to be an exterior algebra on rank $K$ generators and consequently the spectral sequence collapses. The generators lie in $E_{1, *}^{\infty}$. This implies that $\operatorname{Im}[]_{*}$ generates $U_{*}(K, \Gamma)$.

There is a procedure for passing from the homology ring $H_{*}(G)$ to the ring $U_{*}(G)$. It is this: Let $\mu: U_{*}(G) \rightarrow H_{*}(G)$ be the natural transformation defined by $\mu[M, f]=f_{*}\left(\sigma_{M}\right)$ see $[1] . H_{*}(G)$ is $Z\left[w_{1}, w_{2}, \cdots\right.$, $\left.w_{n}\right] / I$ as an algebra where the $w_{i}$ are even dimensional and $I$ is an ideal $\left(f_{1}(w), f_{2}(w), \cdots, f_{k}(w)\right) . f_{i}$ is a homogeneous polynomial in the $w_{i}$. Let $\Gamma \in U_{*}(G)$ be such that $\mu\left(\Gamma_{i}\right)=w_{i}$ and suppose each $\Gamma_{i}$ augments to zero under $U_{*}(G) \rightarrow U_{*}(p t)$. Then $U_{*}(G)=\Lambda\left[\Gamma_{1}, \Gamma_{2}, \cdots\right.$, $\left.\Gamma_{k}\right] / J$ as an algebra where $J$ is the ideal generated by $(*) g_{i}(\Gamma)$ $=f_{i}(\Gamma)+\sum_{j} V_{i j} m_{i j}(\Gamma), i=1 \cdots k$. Here $m_{i j}(\Gamma)$ is a monomial in the $\Gamma_{j}$ 's of total dimension strictly less than that of $f_{i}(\Gamma)$ and $V_{i j} \in \Lambda$. Using the characteristic classes $s_{\omega}[2]$, one can define characteristic numbers $s_{\omega}(\alpha)$ for $\alpha \in U_{*}(G)$ and $\alpha=0$ iff all characteristic numbers $s_{\omega}(\alpha)$ are zero. Since $g_{i}(\Gamma)=0$ we have $s_{\omega}\left(g_{i}(\Gamma)\right)=0$. Expressing this 
via $(*),(* *) s_{\omega}\left(f_{i}(\Gamma)\right)+\sum_{j} s_{\omega}\left(V_{i j} m_{i j}(\Gamma)\right)=0$. Expanding this further gives a sequence of linear equations involving the characteristic numbers $s_{\omega}\left[V_{i j}\right]$ and known quantities. One solves for the $s_{\omega}\left[V_{i j}\right]$ 's which completely determines $V_{i j} \in \Lambda$.

The data necessary to solve the equation $(* *)$ is: (1) A choice of weakly complex manifolds $M_{i}$ and maps $f_{i}: M_{i} \rightarrow G$ such that $\left\{f_{i *}\left(\sigma_{M_{i}}\right)\right\}$ generate the ring $H_{*}(G)$, (2), the ring $H^{*}\left(M_{i}\right),(3)$, the Milnor characteristic classes of $M_{i}$ and the ring homomorphisms $f_{3}^{*}$. Part of this is supplied in [4] and [5]; the remainder by the author.

Having obtained the ring $U_{*}(G, \Gamma)$ one uses homological algebra and determines the algebra $\operatorname{Tor}^{U_{*}(G, \Gamma)}(\Gamma, \Gamma)$ from which the theorems follow.

\section{Bibliography}

1. P. Conner and E. Floyd, Torsion in SU bordism, Mem. Amer. Math. Soc. 60 (1966), $72 \mathrm{pp}$.

2. J. Milnor, Lectures on characteristic classes, Mimeographed notes, Princeton Univ., Princeton, N. J., 1958.

3. S. Araki and $\mathrm{H}$. Toda, Multiplicative structures in $\bmod q$ cohomology theories 1 , Osaka Math J. 2 (1965), 71-115.

4. R. Bott, The space of loops on a Lie group, Michigan Math. J. 5 (1958), 35-61.

5. - Applications of the theory of Morse to symmetric spaces, Amer J. Math. 80 (1958), 965-1029. 436.

6. J. Milnor, Construction of universal bundles. II, Ann. of Math. 63 (1956), 430-

Institute for Defense Analyses 